\title{
Improved Approximation for Orienting Mixed Graphs
}

\author{
Iftah Gamzu* $\quad$ Moti Medina $^{\dagger}$
}

\begin{abstract}
An instance of the maximum mixed graph orientation problem consists of a mixed graph and a collection of source-target vertex pairs. The objective is to orient the undirected edges of the graph so as to maximize the number of pairs that admit a directed source-target path. This problem has recently arisen in the study of biological networks, and it also has applications in communication networks.

In this paper, we identify an interesting local-to-global orientation property. This property enables us to modify the best known algorithms for maximum mixed graph orientation and some of its special structured instances, due to Elberfeld et al. (CPM '11), and obtain improved approximation ratios. We further proceed by developing an algorithm that achieves an even better approximation guarantee for the general setting of the problem. Finally, we study several well-motivated variants of this orientation problem.
\end{abstract}

*Tel Aviv University. Email: iftah.gamzuecs.tau.ac.il.

${ }^{\dagger}$ Tel Aviv University. Email: medinamo@eng.tau.ac.il. 


\section{Introduction}

An instance of the maximum mixed graph orientation problem consists of a mixed graph $G=\left(V, E_{\mathrm{D}} \cup E_{\mathrm{U}}\right)$ with $n$ vertices, such that $E_{\mathrm{D}}$ and $E_{\mathrm{U}}$ indicate the sets of directed and undirected edges, respectively. An additional ingredient of the input is a collection $P \subseteq V \times V$ of source-target vertex pairs. A source-target vertex pair $(s, t) \in P$ is called a request. The objective is to orient $G$ in a way that maximizes the number of satisfied requests. An orientation of $G$ is a directed graph $\vec{G}=\left(V, E_{\mathrm{D}} \cup \overrightarrow{E_{\mathrm{U}}}\right)$, where $\overrightarrow{E_{\mathrm{U}}}$ is a set of directed edges obtained by choosing a single direction for each undirected edge in $E_{\mathrm{U}}$. A request $(s, t)$ is said to be satisfied under an orientation $\vec{G}$ if there is a directed path from $s$ to $t$ in $\vec{G}$.

One may assume without loss of generality that the mixed graph $G$ is acyclic, that is, a graph that has no cycles. This assumption holds since any instance of maximum mixed graph orientation can be reduced to another instance in which the underlying mixed graph is acyclic without affecting the number of requests that can be satisfied $[23,10]$. Indeed, if the input graph contains cycles, one can sequentially contract them one after the other. In each step, the undirected edges of an arbitrary cycle are all oriented in the same direction. In particular, if this cycle contains directed edges then the undirected edges are oriented in a consistent way with those edges. As a result, every pair of vertices on this cycle admits a directed path between them, and thus, the cycle can be contracted. One can easily validate that the resulting mixed acyclic graph consists of undirected components, each of which must be an undirected tree, and those components are connected by directed edges in a way that does not produce cycles. The maximum mixed graph orientation problem draws its interest from applications in network biology and communication networks:

Network biology. Recent technological advances, such as yeast two-hybrid assays [12] and protein coimmunoprecipitation screens [16], enable detecting physical interactions in the cell, leading to protein-protein interaction (PPI) networks. One major caveat of those PPI measurements is that they do not reveal information about the directionality of the interactions, namely, the directions in which the signal flows. Since PPI networks serve as the skeletons of signal transduction in the cell, inferring the hidden directionality information may provide insights to the inner working of the cell. Such an information may be inferred from causal relations in those networks [24]. One such source of causal relations is perturbation experiments, in which a gene is perturbed (cause) and as a result, other genes change their expression levels (effects). A change of expression of a gene suggests that the corresponding proteins admit a path in the network, and in particular, it is assumed that there must be a directed path from the causal gene to the affected gene.

Up until this point in time, the above-mentioned scenario can be modeled as a special instance of the maximum mixed graph orientation problem in which one is interested to orient the edges of an undirected network in a way that maximizes the number of cause-effect pairs that admit a directed path from the causal gene to the affected gene. However, in the more accurate biological variant, there are several interactions whose directionality is known in advance. For instance, protein-DNA interactions are naturally directed from a transcription factor to its regulated genes, and some PPIs, like kinase-substrate interactions, are known to transmit signals in a directional fashion. Therefore, in general, the input network is a mixed graph.

Communication networks. A unidirectional communication network consists of communication links that allow data to travel only in one direction. One main benefit of such communication links is that the data of the device on one side is kept confidential while it may still access the data of the device on the other side. As a consequence, unidirectional networks are most commonly found in high security environments, where a connection may be made between devices with differing security classifications. For example, unidirectional communication links can be used to facilitate access to a vulnerable domain such as the Internet to devices storing sensitive data. The maximum mixed graph orientation problem captures the interesting scenario in which one is interested to design a unidirectional network that maximizes the number of connection requests that can be satisfied in a secure way. We remark that unidirectional networks have also been studied in 
distributed and wireless ad hoc settings (see, e.g., $[2,1,21]$ and the references therein), where a common focus is on algorithmic questions that arise in a given unidirectional network. Here, we are rather interested in the question of how to design such a network while optimizing some performance guarantees.

\subsection{Previous work}

Arkin and Hassin [3] seem to have been the first to study the problem of orienting mixed graphs. They focused on the decision problem corresponding to maximum mixed graph orientation, and demonstrated that it is NPcomplete. Elberfeld et al. [10] observed that the reduction in their proof implies that the maximum mixed graph orientation problem is NP-hard to approximate to within a factor of $7 / 8$. Silverbush, Elberfeld, and Sharan [23] devised a polynomial-size integer linear program formulation for this problem, and evaluated its performance experimentally. Recently, Elberfeld et al. [10] developed several polylogarithmic approximation algorithms for special instances of the problem in which the underlying graph is tree-like, e.g., when the graph has bounded treewidth. In addition, they developed a greedy algorithm for the general setting that achieves $\Omega\left(1 /\left(M^{c} \log n\right)\right)$-approximation, where $M=\max \{n,|P|\}$ and $c=1 / \sqrt{2} \approx 0.7071$.

Medvedovsky et al. [22] initiated the study of the special setting of maximum graph orientation in which the underlying graph is undirected, that is, when there are no pre-directed edges. They proved that it is NP-hard to approximate this problem to within a factor of $12 / 13$, even when the graph is a star. They also proposed an exact dynamic-programming algorithm for the special case of path graphs, and a $\Omega(1 / \log n)$ approximation algorithm for the general problem. Gamzu, Segev and Sharan [15] utilized the framework developed in [14] to obtain an improved $\Omega(\log \log n / \log n)$-approximation ratio (see also [9]). Very recently, Dorn et al. [8] studied this problem from a parameterized complexity point of view. They presented several fixed-parameter tractability results. Further research focused on other variants of this undirected orientation problem. For example, Hakimi, Schmeichel, and Young [17] studied the special setting in which the set of requests contains all vertex pairs, and developed an exact polynomial-time algorithm.

\subsection{Our results}

We identify a useful structural property of requests crossing through a junction vertex. Informally, this property guarantees that if a set of requests is locally satisfiable then it can also be satisfied globally. Using this property, we can slightly modify the algorithms developed by Elberfeld et al. [10], and obtain improved approximation ratios. For example, we eliminate a logarithmic factor from their polylogarithmic approximation ratio for the case that the underlying graph has bounded treewidth. These results appear in Section 2. Although the local-to-global property can be used in conjunction with the algorithm of Elberfeld et al. [10] to obtain an improved approximation guarantee for the general setting, we proceed by developing an improved

$\Omega\left(1 /(n|P|)^{1 / 3}\right)$-approximation algorithm for this problem. Our algorithm is based on a greedy approach that employs the local-to-global property in a novel way. The specifics of this algorithm are presented in Section 3. We also study two well-motivated variants of the orientation problem, and most notably, show hardness results for them. Further details are provided in Section 4.

\section{From Local to Global Orientations}

In this section, we identify a useful structural property of requests crossing through a junction vertex. Informally, this property guarantees that if there is an orientation of the local neighborhood of a vertex that locally satisfies a set of requests then it can be extended to a global orientation of the complete graph which satisfies the same set of requests. Finding a local orientation that maximizes the local satisfiability is a relatively easy task, namely, it admits a constant factor approximation algorithm. As a consequence, we can slightly modify 
the algorithms developed by Elberfeld et al. [10] so they utilize this property, and obtain improved approximation ratios. For example, we eliminate a logarithmic factor from their polylogarithmic approximation ratio for the special case that the underlying graph has bounded treewidth.

We associate each request $(s, t) \in P$ with the shortest path $p$ between $s$ and $t$ in the underlying graph. Note that in case there are several shortest paths for a request, we associate it with one of them arbitrarily. We now introduce some notation and terminology. To better understand the suggested notation, we refer the reader to the concrete example in Figure 1.

- The local neighborhood of a vertex $v$ is the subgraph $G_{v}$ that consists of $v$, all edges incident on $v$, and all vertices adjacent to $v$. Notice that the local neighborhood graph is a star.

- Let $P_{v}$ be the set of shortest paths of requests that cross $v$, and let $P_{v}^{\prime}$ be the corresponding set of local paths, that is, the paths of $P_{v}$ confined to the local neighborhood of $v$. More precisely, each (global) path $p \in P_{v}$ gives rise to a (local) path $p^{\prime} \in P_{v}^{\prime}$ defined as the intersection of $p$ with the local neighborhood of $v$. Furthermore, for each $p^{\prime} \in P_{v}^{\prime}$, we define its local endpoints $s^{\prime}$ and $t^{\prime}$ to be the closest vertices to $s$ and $t$ on $p$ that also appear on $p^{\prime}$, respectively.

- The local graph orientation problem corresponding to vertex $v$ is defined with respect to the local neighborhood graph $G_{v}$ and the set of local paths $P_{v}^{\prime}$. The goal is to orient the undirected edges of $G_{v}$ in a way that maximizes the number of satisfied paths in $P_{v}^{\prime}$. A path is said to be satisfied if there is a directed path between its source and target vertices under the orientation.

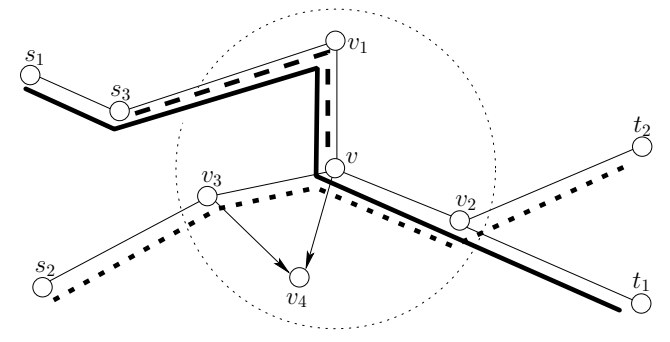

(a)

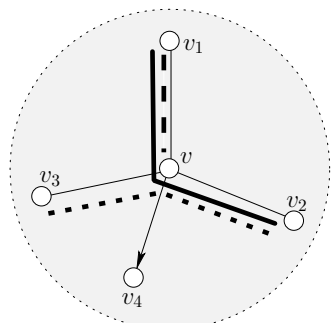

(b)

Figure 1: (a) Suppose $P=\left\{\left(s_{1}, t_{1}\right),\left(s_{2}, t_{2}\right),\left(s_{3}, v\right)\right\}$ is the set of requests, and note that the shortest paths of these requests are marked with the heavy lines. Notice that all these paths cross $v$. (b) The local neighborhood of $v$, and the corresponding set of local paths. For example, notice that the local endpoints of the request $\left(s_{1}, t_{1}\right)$ are $s_{1}^{\prime}=v_{1}$ and $t_{1}^{\prime}=v_{2}$.

Lemma 2.1. Given an orientation of $G_{v}$ that satisfies a set of local paths $S^{\prime} \subseteq P_{v}^{\prime}$ then there is an orientation of $G$ that satisfies the corresponding set of global paths $S \subseteq P_{v}$.

Proof. We argue that if two local paths $p_{1}^{\prime}, p_{2}^{\prime} \in S^{\prime}$ then the corresponding global paths $p_{1}, p_{2} \in S$ cannot be in conflict. The paths $p_{1}$ and $p_{2}$ are said to be in conflict if they have a mutual undirected edge that gets a different direction when the edges of $p_{1}$ are consistently oriented from its source vertex to its target vertex and when the edges of $p_{2}$ are consistently oriented from its source vertex to its target vertex. Notice that establishing this argument completes that proof of the lemma since none of the paths of $S$ can be in conflict with another path in $S$, and therefore, all the paths in $S$ can be simultaneously satisfied by consistently orienting each one of them from its source vertex to its target vertex. Note that after one orients those paths, the remaining undirected edges of the graph can be oriented in some arbitrary way. 
For the purpose of establishing the above argument, let us suppose that $p_{1}$ and $p_{2}$ are in conflict, and attain a contradiction. Since $p_{1}$ and $p_{2}$ are in conflict then there is an undirected edge $e=\left(v_{1}, v_{2}\right) \in E_{\mathrm{U}}$ that gets a different direction when consistently orienting each one of $p_{1}$ and $p_{2}$ from its source vertex to its target vertex. Let us assume without loss of generality that edge $e$ is the closest to $v$ from all conflicting edges. We next present a case analysis that depends whether the edge $e$ appears before or after the position of vertex $v$ on each of paths $p_{1}$ and $p_{2}$. Essentially, there are two main cases. To better understand the used notation, we refer the reader to the concrete examples in Figure 2.

Case I: edge $e$ appears after vertex $\boldsymbol{v}$ in both $\boldsymbol{p}_{\mathbf{1}}$ and $\boldsymbol{p}_{2}$. Let us assume without loss of generality that $v_{1}$ is closer to $v$ than $v_{2}$ on $p_{1}$, and $v_{2}$ is closer to $v$ than $v_{1}$ on $p_{2}$. Let $d_{1}$ be the distance between $v$ and $v_{1}$ on $p_{1}$, and $d_{2}$ be the distance between $v$ and $v_{2}$ on $p_{2}$. Since $p_{1}$ is a shortest path between $s_{1}$ and $t_{1}$, it must also be a shortest path between $v$ and $v_{2}$. Thus, $d_{1}+1 \leq d_{2}$. Similarly, since $p_{2}$ is a shortest path between $s_{2}$ and $t_{2}$, it must also be a shortest path between $v$ and $v_{1}$, and hence, $d_{2}+1 \leq d_{1}$. Summing together the above inequalities results in $d_{1}+d_{2}+2 \leq d_{1}+d_{2}$, a contradiction.

We note that the case that the edge $e$ appears before vertex $v$ in both $p_{1}$ and $p_{2}$ can be handled along the same lines with an adjustment to the relative position of $v$, e.g., the distances need to be defined from $v_{1}$ and $v_{2}$ towards the junction vertex $v$.

Case II: edge $e$ appears after vertex $v$ in $p_{1}$ and before vertex $v$ in $p_{2}$. Let us assume without loss of generality that $v_{1}$ is closer to $v$ than $v_{2}$ on both paths $p_{1}$ and $p_{2}$. Since $p_{1}^{\prime}, p_{2}^{\prime} \in S^{\prime}$ we know that the edge on which $p_{1}$ leaves $v$ and the edge on which $p_{2}$ enters $v$ must be different. This implies that the subpath between $v$ and $v_{1}$ on $p_{1}$ and the subpath between $v_{1}$ and $v$ on $p_{2}$ are different. Consequently, merging these two subpaths creates a cycle in the graph. This contradicts the fact that the graph is acyclic.

Note that the case that the edge $e$ appears after vertex $v$ in $p_{2}$ and before vertex $v$ in $p_{1}$ is essentially identical to the above case up to a renaming of the paths.

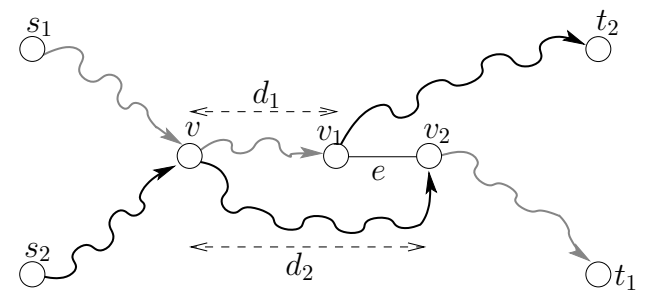

(a)

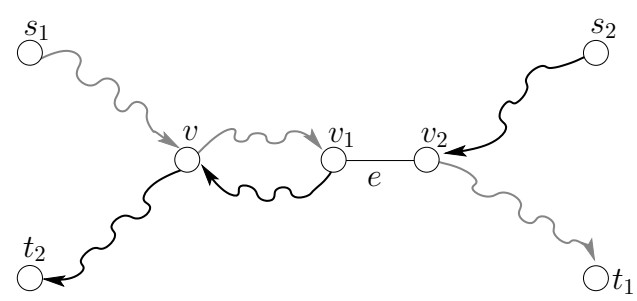

(b)

Figure 2: (a) The case that $e$ appears after $v$ in both $p_{1}$ and $p_{2}$. (b) The case that $e$ appears after $v$ in $p_{1}$ and before $v$ in $p_{2}$.

We now concentrate on the computational complexity of the local graph orientation problem corresponding to a vertex $v$. One can easily validate that this problem is equivalent to the maximum undirected graph orientation problem on a star. Medvedovsky et al. [22] demonstrated that this problem is equivalent to the maximum directed cut problem. This latter problem admits constant factor approximation algorithms (see, e.g., $[11,20])$. In fact, one can easily verify that a random orientation of the undirected edges in the local neighborhood satisfies at least $1 / 4$ of the paths of $P_{v}^{\prime}$ in expectation. This follows since the maximal length of any path in the local neighborhood is at most 2. Furthermore, one can use the method of conditional expectations to obtain a deterministic orientation that satisfies at least $1 / 4$ of the paths, and consequently, this approach is a 1/4-approximation for this problem. Combining this result with the local-to-global orientation property exhibited in Lemma 2.1 implies the following theorem. 
Theorem 2.2. Given a vertex $v$ and a set of requests $P_{v}$ whose shortest paths cross $v$, there is a polynomialtime algorithm that computes an orientation that satisfies $\Omega\left(\left|P_{v}\right|\right)$ requests.

We can now modify the algorithms developed by Elberfeld et al. [10] in accordance with Theorem 2.2, and obtain the following improved approximation ratios. We emphasize that the algorithms and their analysis follow (up to our modification step) those presented by Elberfeld et al. [10], and thus, we defer them to the appendix. The first two theorems present algorithms whose approximation guarantees depend on the treewidth and feedback vertex number of the underlying graph.

Theorem 2.3. There is a polynomial-time algorithm that finds an orientation satisfying $\Omega(|P| /(k \log n))$ requests when the undirected version of the underlying graph has bounded treewidth $k$.

Theorem 2.4. There is a polynomial-time algorithm that finds an orientation satisfying $\Omega(|P| /(k+\log n))$ requests, where $k$ is the minimum number of vertices whose deletion turns the undirected version of the underlying graph into a tree.

We can also improve the approximation ratios of the algorithms presented by Elberfeld et al. [10] for the general case, in which there are no structural assumptions on the graph, by a logarithmic factor.

Theorem 2.5. There is a polynomial-time algorithm that approximates the maximum mixed graph orientation problem to within a factor of $\Omega(1 / \sqrt{\Delta|P|})$, where $\Delta$ is the maximum length of a shortest source-target path in the graph.

Theorem 2.6. There is a polynomial-time algorithm that approximates the maximum mixed graph orientation problem to within a factor of $\Omega\left(1 / M^{1 / \sqrt{2}}\right)$, where $M=\max \{n,|P|\}$.

Note that we do not provide a proof for the latter theorem since it can be established along the same lines of [10], but more importantly, since we next present an algorithm with a better approximation guarantee.

\section{Improved Approximation for The General Case}

In this section, we develop a relatively simple $\Omega\left(1 /(n|P|)^{1 / 3}\right)$-approximation algorithm for the maximum mixed graph orientation problem. Our algorithm is based on a greedy approach that employs the local-toglobal orientation property developed in Section 2.

The algorithm, formally described below, begins by associating each request $\left(s_{i}, t_{i}\right) \in P$ with a shortest path $p_{i}$ between $s_{i}$ and $t_{i}$ in the graph. Then, it greedily orients shortest paths one after the other until all the remaining paths are in conflict with many other paths. When this happens, the algorithm concentrates on the vertex that is crossed by a maximal number of paths, and utilizes the local-to-global orientation algorithm from Theorem 2.2 to complete the orientation of the graph. Recall that two paths $p_{1}$ and $p_{2}$ are said to be in conflict if they have a mutual undirected edge that gets a different direction when the edges of $p_{1}$ and $p_{2}$ are consistently oriented from their source vertex to their target vertex.

One can easily verify that the algorithm computes a feasible orientation, namely, it assigns a single direction to each undirected edge. This follows since no conflicting paths are oriented during the main loop of the algorithm, and since the algorithm from Theorem 2.2 is known to compute a feasible orientation. We next prove that the algorithm satisfies $\Omega\left(1 /(n|P|)^{1 / 3}\right)$-fraction of all requests. Clearly, this implies that the algorithm achieves (at least) the same approximation guarantee.

Theorem 3.1. The greedy orientation algorithm satisfies $\Omega\left(1 /(n|P|)^{1 / 3}\right)$-fraction of all requests. 


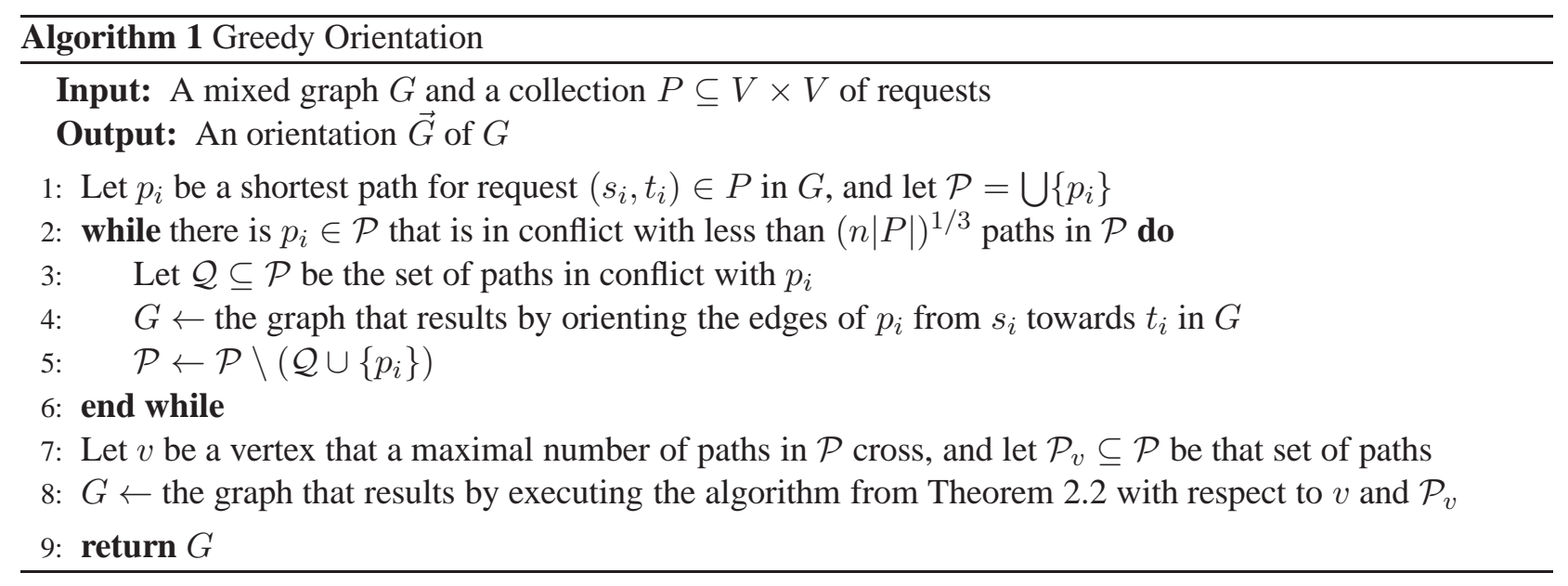

Proof. Let $\mathcal{P}=\bigcup\left\{p_{i}\right\}$ be the initial collection of shortest paths, and note that $|\mathcal{P}|=|P|$. In addition, let $\mathcal{P}_{2} \subseteq \mathcal{P}$ be the set of paths the remain after the termination of the main loop of the algorithm, and $\mathcal{P}_{1}=\mathcal{P} \backslash \mathcal{P}_{2}$. Finally, let $\mathcal{A}_{1}$ be the set of paths that our algorithm satisfies during the main loop of the algorithm, and let $\mathcal{A}_{2}$ be the set of paths that the algorithm satisfies during the execution of the algorithm from Theorem 2.2. In what follows, we prove that $\left|\mathcal{A}_{1}\right|=\Omega\left(1 /(n|P|)^{1 / 3}\right) \cdot\left|\mathcal{P}_{1}\right|$, and $\left|\mathcal{A}_{2}\right|=\Omega\left(1 /(n|P|)^{1 / 3}\right) \cdot\left|\mathcal{P}_{2}\right|$. Consequently, we obtain that the number of paths satisfied by our algorithm is

$$
\left|\mathcal{A}_{1}\right|+\left|\mathcal{A}_{2}\right|=\Omega\left(\frac{1}{(n|P|)^{1 / 3}}\right) \cdot\left(\left|\mathcal{P}_{1}\right|+\left|\mathcal{P}_{2}\right|\right)=\Omega\left(\frac{1}{(n|P|)^{1 / 3}}\right) \cdot|P|
$$

The fact that $\left|\mathcal{A}_{1}\right|=\Omega\left(1 /(n|P|)^{1 / 3}\right) \cdot\left|\mathcal{P}_{1}\right|$ easily follows by observing that in each step of the main loop of the algorithm, one path is satisfied while less than $(n|P|)^{1 / 3}$ paths are discarded. Hence, we are left to prove that $\left|\mathcal{A}_{2}\right|=\Omega\left(1 /(n|P|)^{1 / 3}\right) \cdot\left|\mathcal{P}_{2}\right|$. We establish a somewhat stronger result by demonstrating that $\left|\mathcal{A}_{2}\right|=\Omega\left(1 /\left(n\left|\mathcal{P}_{2}\right|\right)^{1 / 3}\right) \cdot\left|\mathcal{P}_{2}\right|$. For this purpose, consider two paths $p_{1}, p_{2} \in \mathcal{P}_{2}$ that are in conflict. We associate the conflict between these paths to an arbitrary undirected edge that gets a different direction when $p_{1}$ and $p_{2}$ are oriented, and place one token on this edge. Notice that each path of $\mathcal{P}_{2}$ is in conflict with at least $(n|P|)^{1 / 3}$ other paths in $\mathcal{P}_{2}$; otherwise, the main loop would not have terminated. This implies that if we place a token for each pair of conflicting paths in $\mathcal{P}_{2}$ as shown before then the undirected edges of $G$ have at least $(n|P|)^{1 / 3} \cdot\left|\mathcal{P}_{2}\right| / 2 \geq n^{1 / 3}\left|\mathcal{P}_{2}\right|^{4 / 3} / 2$ tokens placed on them. As a consequence, there must be a vertex that has at least $t=\left|\mathcal{P}_{2}\right|^{4 / 3} /\left(2 n^{2 / 3}\right)$ tokens placed on the undirected edges in its local neighborhood. We next argue that if some vertex has $t$ tokens in its local neighborhood then there must be $\Omega(\sqrt{t})$ paths that cross that vertex. As a result, we attain that the number of paths that cross the vertex $v$, i.e., the vertex that a maximal number of paths from $\mathcal{P}_{2}$ cross, is at least $\Omega(\sqrt{t})=\Omega\left(\left|\mathcal{P}_{2}\right|^{2 / 3} / n^{1 / 3}\right)$. By theorem 2.2, our algorithm satisfies a constant fraction of these requests, namely, $\left|\mathcal{A}_{2}\right|=\Omega\left(1 /\left(n\left|\mathcal{P}_{2}\right|\right)^{1 / 3}\right) \cdot\left|\mathcal{P}_{2}\right|$, as required.

For the purpose of establishing the above argument, consider some vertex $u$ that has $t$ tokens in its local neighborhood. Let us focus on some edge $e$ in this local neighborhood that has $r$ paths that traverse in one direction and $\ell$ paths that traverse in the other direction. Notice that such an edge is assigned $r \cdot \ell$ tokens. This implies that if the local neighborhood of $u$ consists only of the edge $e$ then the minimal number of paths that cross $u$ corresponds to the solution of $\min \{r+\ell: r \cdot \ell=t\}$. One can easily verify that the solution for this expression is $r=\ell=\sqrt{t}$, that is, the number of paths is $\Omega(\sqrt{t})$. Note that when there is more than one edge in the local neighborhood of $u$ then any path may cross at most two edges. As a result, if we denote the set of edges in the local neighborhood of $u$ by $E_{u}$, then the minimal number of paths that cross $u$ dominates the solution of $\min \left\{\sum_{e \in E_{u}}\left(r_{e}+\ell_{e}\right) / 2: \sum_{e}\left(r_{e} \cdot \ell_{e}\right)=t\right\}$; here, $r_{e}$ and $\ell_{e}$ indicate the number of paths traversing 
edge $e$ in one direction and the other direction, respectively. One can easily demonstrate that the solution for the above expression is obtained by assigning non-zero values only to one pair of $r_{e}, \ell_{e}$ variables, namely, it is equivalent to the solution for the single edge case.

\section{Other Orientation Variants}

In this section, we study two well-motivated variants of the orientation problem: the first is maximum mixed graph orientation with fixed paths, and the other is maximum mixed grid orientation.

\subsection{Orientation with fixed paths}

We consider the maximum mixed graph orientation with fixed paths problem. This variant is identical to the maximum mixed graph orientation problem with the exception that each request $(s, t) \in P$ is also associated with a fixed path $p$ from $s$ to $t$ in the graph. With this modified definition in mind, a request $(s, t)$ is satisfied only if the edges of the path $p$ are oriented from the vertex $s$ towards the vertex $t$. Note that this variant is seemingly simpler than maximum mixed graph orientation since the only computational task is to decide which requests to satisfy, and there is no need to decide which paths will be used to satisfy those requests. This is also one of our motivations for studying this variant, hoping that it will shed some light on the original problem that would lead to a reduction in the gap between its lower and upper approximation bounds.

We prove that the maximum mixed graph orientation with fixed paths problem is NP-hard to approximate to within a factor of $\max \left\{1 /|P|^{1-\epsilon}, 1 / m^{1 / 2-\epsilon}\right\}$, for any $\epsilon>0$. In fact, we establish this result even when the underlying graph is undirected. As a consequence, we attain that this problem is provably harder than the maximum mixed (or undirected) graph orientation problem, although it may seem simpler at first glance. Our proof is based on showing that the problem under consideration captures the well-known maximum independent set problem as a special case.

A hardness of approximation result. An input instance for the maximum independent set problem consists of an undirected graph $G^{\prime}=\left(V^{\prime}, E^{\prime}\right)$. The goal is to find an independent set of maximum size in the graph. An independent set is a collection of vertices that do not have any edges between them. This problem is known to be NP-hard to approximate within a factor of $1 /\left|V^{\prime}\right|^{1-\epsilon}$, for any $\epsilon>0$ [25]. We next show a value-preserving reduction from this problem to our maximum mixed graph orientation with fixed paths problem.

Given an input instance of maximum independent set, we construct an input instance for our problem that consists of the undirected graph presented in Figure 3(a). Specifically, we begin by creating a graph with $n^{\prime}=\left|V^{\prime}\right|$ pairs of $s_{i}, t_{i}$ vertices, corresponding to the vertices of $G^{\prime}$, such that each such pair is connected by a path $p_{i}$. We intersect all these connecting paths in a grid-like fashion. Then, each intersection point is replaced by one of the gadgets exhibited in Figures 3(b) and 3(c). The gadget $g_{i, j}$ that replaces the intersection point of paths $p_{i}$ and $p_{j}$ has 4 vertices: $v_{i}$ and $u_{i}$ that are appropriately added to path $p_{i}$, and $v_{j}$ and $u_{j}$ that are appropriately added to path $p_{j}$. The edges within the gadget has the form described in Figure 3(b) if $(i, j) \notin E^{\prime}$, or the form described in Figure 3(c) if $(i, j) \in E^{\prime}$. In the latter case, the path $p_{j}$ is also modified to consist of the vertices $v_{i}$ and $u_{i}$, so its subpath inside the gadget is $\left\langle v_{j}, u_{i}, v_{i}, u_{j}\right\rangle$. In addition, the set of requests $P$ for our problem consist of all $n^{\prime}$ pairs $\left(s_{i}, t_{i}\right)$ with their corresponding path $p_{i}$.

One can easily validate that a solution $S \subseteq V^{\prime}$ for the maximum independent set problem implies an orientation in the newly-created instance that satisfies the same number of requests. Specifically, if $i, j \in S$ then clearly $(i, j) \notin E^{\prime}$, and thus, the paths $p_{i}$ and $p_{j}$ do not share edges. As a result, one can simultaneously satisfy both request $\left(s_{i}, t_{i}\right)$ and $\left(s_{j}, t_{j}\right)$ by orienting each of their paths from its source vertex to its target vertex. Conversely, it is not difficult to verify that given an orientation in newly-created instance that satisfies some set of requests, one can perform a similar value-preserving transformation in the opposite direction. In 


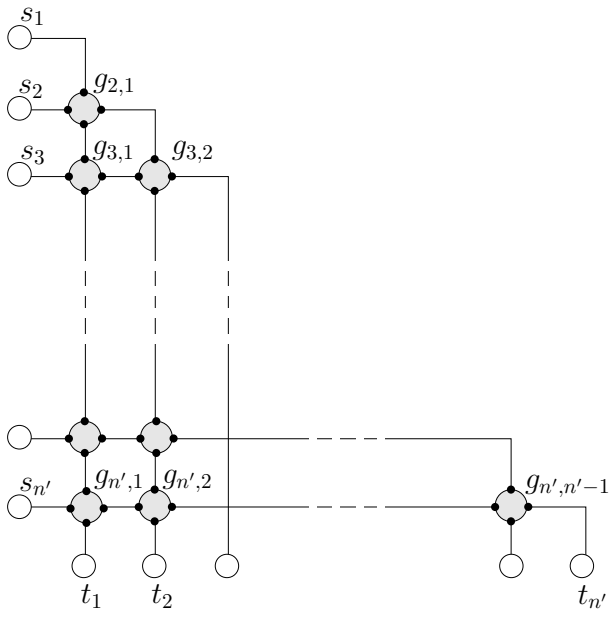

(a)

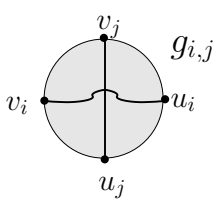

(b)

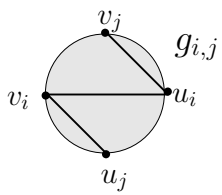

(c)

Figure 3: (a) The graph resulting from the reduction. (b) The gadget that is used in case $(i, j) \notin E^{\prime}$. Note that the edges $\left(v_{i}, u_{i}\right)$ and $\left(v_{j}, u_{j}\right)$ do not intersect. (c) The gadget that is used in case $(i, j) \in E^{\prime}$.

particular, notice that if $i \notin S$ then there must be $j \in S$ such that $(i, j) \in E^{\prime}$, and hence, the paths $p_{i}$ and $p_{j}$ require to orient the edge $\left(v_{i}, u_{i}\right)$ in the gadget $g_{i, j}$ in conflicting directions. Consequently, one cannot simultaneously satisfy both underlying requests.

As a result of this value-preserving reduction, and in conjunction with the hardness result presented by Zuckerman [25], we attain the following inapproximability result. Recall that $|P|=\left|V^{\prime}\right|$, and notice that the number of edges in the newly-created instance is $m=O\left(\left|V^{\prime}\right|^{2}\right)$.

Theorem 4.1. The maximum mixed graph orientation with fixed paths problem is NP-hard to approximate within a factor of $\max \left\{1 /|P|^{1-\epsilon}, 1 / m^{1 / 2-\epsilon}\right\}$, for any $\epsilon>0$.

\subsection{Orientation in grid networks}

We study the maximum mixed grid orientation problem. This variant is identical to the maximum mixed graph orientation problem with the additional restriction that the graph is a grid. A $n \times m$ grid network is a graph with a vertex set $V=\{1, \ldots, n\} \times\{1, \ldots, m\}$, and an edge set $E$ consisting of horizontal edges, i.e., edges $((i, j),(i, j+1))$ for all $j=\{1, \ldots, m-1\}$, and vertical edges, i.e., edges $((i, j),(i+1, j))$ for all $i=\{1, \ldots, n-1\}$. Note that the study of this variant is motivated by applications in networking.

We prove that the maximum mixed grid orientation problem is at least as hard as the maximum directed cut problem. Consequently, approximating our problem within factors of $12 / 13 \approx 0.923$ and $\alpha_{\mathrm{GW}} \approx 0.878$ is NP-hard and Unique Game-hard, respectively. Interestingly, this finding comes in contrast with the results attainable for the undirected grid setting. This latter setting can be solved to optimality in polynomial-time, and in particular, when the grid is not a path, that is, when $n, m>1$, all the requests in $P$ can be satisfied.

A hardness of approximation result. An input instance for the maximum directed cut problem consists of a directed graph $G^{\prime}=\left(V^{\prime}, E^{\prime}\right)$. The goal is to find a directed cut of maximum size in the graph. The size of a cut $A \subseteq V$ is the number of directed edges $(u, v) \in E^{\prime}$ such that $u \in A$ and $v \in V^{\prime} \backslash A$. Approximating this problem within factors of $12 / 13 \approx 0.923$ and $\alpha_{\mathrm{GW}} \approx 0.878$ is known to be NP-hard [18] and Unique Games-hard [19], respectively. In what follows, we present a value-preserving reduction from this problem to our maximum mixed grid orientation problem. 
Given an input instance of maximum directed cut, we construct an input instance for our problem which consists of the mixed grid presented in Figure 4(a). Specifically, we create a grid whose dimensions are $n=2\left|V^{\prime}\right|-1$ and $m=3$. We associate each vertex $v_{i} \in V^{\prime}$ with the vertex $(2 i-1,1)$ in the grid. The edges incident on each vertex $(2 i, 1)$ in the grid are oriented away from that vertex, and the edges along the perimeter of the sub-grid that consists of the second and third vertex columns are oriented in a way that creates a directed cycle. In addition, the set of requests for our problem is defined to be $P=E^{\prime}$.

One can validate that a solution $A \subseteq V^{\prime}$ for the maximum directed cut problem implies an orientation in the newly-created instance that satisfies the same number of requests. Specifically, if $v_{i} \in A$ we orient the single undirected edge incident on vertex $v_{i}$ of the grid away from that vertex, and if $v_{i} \in V \backslash A$ we orient that edge towards vertex $v_{i}$. Then, it is easy to see that if an edge of $E^{\prime}$ is cut by the solution $A$ then the corresponding request is satisfied in the orientation. Conversely, it is not difficult to verify that given an orientation in newly-created instance that satisfies some set of requests, one can perform a similar value-preserving transformation in the opposite direction. In particular, this side of the proof builds upon the observation that any request $\left(v_{i}, v_{j}\right)$ may only be satisfied by a path that crosses the undirected edges incident on $v_{i}$ and $v_{j}$. The orientation of all those undirected edges define the cut in the initial problem.

As a result of this value-preserving reduction, and in conjunction with the hardness results presented by Håstad [18] and Khot et al. [19], we attain the following inapproximability result.

Theorem 4.2. The maximum mixed grid orientation problem is NP-hard to approximate within a factor of $12 / 13 \approx 0.923$, and Unique Games-hard to approximate within a factor of $\alpha_{\mathrm{GW}} \approx 0.878$.

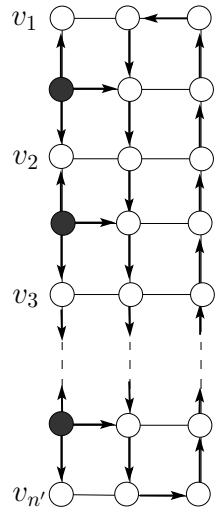

(a)

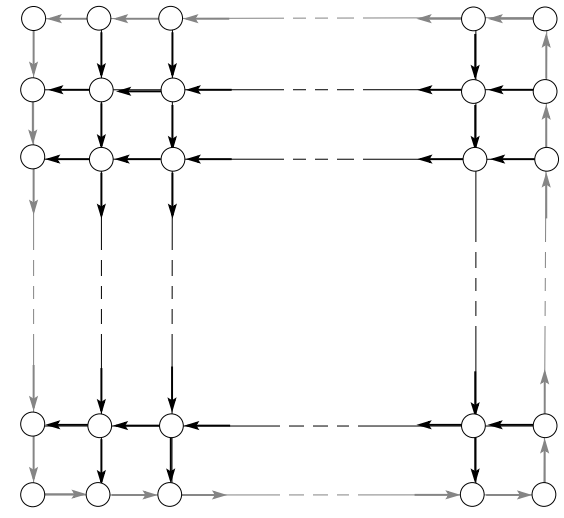

(b)

Figure 4: (a) The grid resulting from the reduction. Note that $n^{\prime}=\left|V^{\prime}\right|$. (b) An orientation of an undirected grid that admits a directed path between any two vertices.

Orientation of undirected grids. The above-mentioned hardness result comes in contrast with the results attainable for the undirected grid setting. This latter setting can be solved to optimality in polynomial-time. Specifically, when the grid is a path, i.e., when either $m$ or $n$ equals 1 , there are optimal polynomial-time algorithms for the problem [22,8], and when $n, m>1$, there is a simple orientation that satisfies all the requests in $P$. This orientation can be obtained by creating a directed cycle along the perimeter of the grid, and then, orienting all the remaining horizontal and vertical edges consistently. A concrete example of such an orientation is presented in Figure 4(b). One can easily prove that this orientation admits a directed path between any two vertices of the graph. 


\section{References}

[1] Y. Afek and A. Bremler-Barr. Self-stabilizing unidirectional network algorithms by power supply. Chicago J. Theor. Comput. Sci., 1998.

[2] Y. Afek and E. Gafni. Distributed algorithms for unidirectional networks. SIAM J. Comput., 23(6):1152$1178,1994$.

[3] E. M. Arkin and R. Hassin. A note on orientations of mixed graphs. Discrete Applied Mathematics, 116(3):271-278, 2002.

[4] V. Bafna, P. Berman, and T. Fujito. A 2-approximation algorithm for the undirected feedback vertex set problem. SIAM J. Discrete Math., 12(3):289-297, 1999.

[5] A. Becker and D. Geiger. Approximation algorithms for the loop cutset problem. In Proceedings 10th Annual Conference on Uncertainty in Artificial Intelligence, pages 60-68, 1994.

[6] H. L. Bodlaender. A linear-time algorithm for finding tree-decompositions of small treewidth. SIAM J. Comput., 25(6):1305-1317, 1996.

[7] F. A. Chudak, M. X. Goemans, D. S. Hochbaum, and D. P. Williamson. A primal-dual interpretation of two 2-approximation algorithms for the feedback vertex set problem in undirected graphs. Oper. Res. Lett., 22(4-5):111-118, 1998.

[8] B. Dorn, F. Hüffner, D. Krüger, R. Niedermeier, and J. Uhlmann. Exploiting bounded signal flow for graph orientation based on cause-effect pairs. In Proceedings 1st International ICST Conference on Theory and Practice of Algorithms in (Computer) Systems, pages 104-115, 2011.

[9] M. Elberfeld, V. Bafna, I. Gamzu, A. Medvedovsky, D. Segev, D. Silverbush, U. Zwick, and R. Sharan. On the approximability of reachability-preserving network orientations. Internet Mathematics, 7:209232, 2011.

[10] M. Elberfeld, D. Segev, C. R. Davidson, D. Silverbush, and R. Sharan. Approximation algorithms for orienting mixed graphs. In Proceedings 22nd Annual Symposium on Combinatorial Pattern Matching, pages 416-428, 2011.

[11] U. Feige and M. X. Goemans. Aproximating the value of two prover proof systems, with applications to MAX 2SAT and MAX DICUT. In Proceedings 3rd Israel Symposium on Theory and Computing Systems, pages 182-189, 1995.

[12] S. Fields. High-throughput two-hybrid analysis: The promise and the peril. The FEBS Journal, 272(21):5391-5399, 2005.

[13] G. N. Frederickson and D. B. Johnson. Generating and searching sets induced by networks. In Proceedings 7th International Colloquium on Automata, Languages and Programming, pages 221-233, 1980.

[14] I. Gamzu and D. Segev. A sublogarithmic approximation for highway and tollbooth pricing. In Proceedings 37th International Colloquium on Automata, Languages and Programming, pages 582-593, 2010. 
[15] I. Gamzu, D. Segev, and R. Sharan. Improved orientations of physical networks. In Proceedings 10th International Workshop on Algorithms in Bioinformatics, pages 215-225, 2010.

[16] A. C. Gavin, M. Bosche, R. Krause, P. Grandi, M. Marzioch, A. Bauer, J. Schultz, J. M. Rick, A. M. Michon, C. M. Cruciat, M. Remor, C. Hofert, M. Schelder, M. Brajenovic, H. Ruffner, A. Merino, K. Klein, M. Hudak, D. Dickson, T. Rudi, V. Gnau, A. Bauch, S. Bastuck, B. Huhse, C. Leutwein, M. A. Heurtier, R. R. Copley, A. Edelmann, E. Querfurth, V. Rybin, G. Drewes, M. Raida, T. Bouwmeester, P. Bork, B. Seraphin, B. Kuster, G. Neubauer, and G. Superti-Furga. Functional organization of the yeast proteome by systematic analysis of protein complexes. Nature, 415:141-147, 2002.

[17] S. L. Hakimi, E. F. Schmeichel, and N. E. Young. Orienting graphs to optimize reachability. Information Processing Letters, 63(5):229-235, 1997.

[18] J. Håstad. Some optimal inapproximability results. Journal of the ACM, 48(4):798-859, 2001.

[19] S. Khot, G. Kindler, E. Mossel, and R. O'Donnell. Optimal inapproximability results for MAX-CUT and other 2-variable CSPs? SIAM Journal on Computing, 37(1):319-357, 2007.

[20] M. Lewin, D. Livnat, and U. Zwick. Improved rounding techniques for the MAX 2-SAT and MAX DI-CUT problems. In Proceedings 9th International Conference on Integer Programming and Combinatorial Optimization, pages 67-82, 2002.

[21] M. K. Marina and S. R. Das. Routing performance in the presence of unidirectional links in multihop wireless networks. In Proceedings 3rd ACM Interational Symposium on Mobile Ad Hoc Networking and Computing, pages 12-23, 2002.

[22] A. Medvedovsky, V. Bafna, U. Zwick, and R. Sharan. An algorithm for orienting graphs based on cause-effect pairs and its applications to orienting protein networks. In Proceedings 8th International Workshop on Algorithms in Bioinformatics, pages 222-232, 2008.

[23] D. Silverbush, M. Elberfeld, and R. Sharan. Optimally orienting physical networks. In Proceedings 15th Annual International Conference on Research in Computational Molecular Biology, pages 424 436, 2011.

[24] C.-H. Yeang, T. Ideker, and T. Jaakkola. Physical network models. Journal of Computational Biology, 11(2/3):243-262, 2004.

[25] D. Zuckerman. Linear degree extractors and the inapproximability of max clique and chromatic number. Theory of Computing, 3:103-128, 2007.

\section{A Additional Details}

In this section, we complete the details omitted from the main part of the paper.

\section{A.1 Proof of Theorem 2.3}

The algorithm begins by computing a tree decomposition of width $k$ for the undirected version of the underlying graph. Note that this task can be done in polynomial-time since our graph has bounded treewidth [6]. A tree decomposition is a pair $(T, X)$, where $X=\left\{X_{1}, \ldots, X_{\ell}\right\}$ is a collection of subsets such that each 
$X_{i} \subseteq V$, and $T$ is a tree whose nodes are the subsets in $X$. Note that $\ell=\operatorname{poly}(n)$ in our case. The decomposition satisfies the following properties: (1) $\bigcup X_{i}=V$, (2) the incident vertices of every edge of the graph are contained in some subset in $X$, and (3) if $X_{i}$ and $X_{j}$ contain a vertex $v$ then all the nodes $X_{k}$ in the unique path between $X_{i}$ and $X_{j}$ contain $v$ as well. The width of the tree is defined to be $\max \left|X_{i}\right|-1$.

Given the tree decomposition $(T, X)$, the algorithm proceeds by computing a shortest path for each request in $P$. The paths are then classified into at most $\lceil\log \ell\rceil=O(\log n)$ classes such that for every class, an orientation that satisfies $\Omega(1 / k)$-fraction of its paths can be efficiently computed. As a consequence, by separately computing an orientation for each class, and then picking the option that satisfies the highest number of paths, we are guaranteed to satisfy $\Omega(|P| /(k \log n))$ of all the requests.

For the purpose of constructing the first class, we find a centroid node $X_{t}$ of $T$, that is, a node whose removal breaks the tree into a collection of subtrees, each of which has at most half of the vertices in $T$. Note that any tree has a centroid (see, e.g., [13]). We assign all the paths that cross a vertex from $X_{t}=\left\{v_{1}, \ldots, v_{r}\right\}$ to class $\mathcal{C}_{1}$. We further partition $\mathcal{C}_{1}$ into $r$ collections $\mathcal{C}_{1,1}, \ldots, \mathcal{C}_{1, r}$ such that a path $p$ is assigned to the collection $\mathcal{C}_{1, j}$ if it crosses $v_{j}$ but does not cross any of the vertices in $\left\{v_{1}, \ldots, v_{j-1}\right\}$. Notice that we can satisfy $\Omega\left(\left|\mathcal{C}_{1, j}\right|\right)$ paths from the collection $j$ by applying Theorem 2.2. One can now easily validate that executing the mentioned algorithm on each collection separately, and then picking the option that satisfies the highest number of paths results in an orientation satisfying $\Omega\left(\left|\mathcal{C}_{1}\right| / k\right)$ requests since $r \leq k+1$.

To construct the second class, we first remove the node $X_{t}$ from $T$ to obtain a forest of tree decompositions. For each tree decomposition, we compute a centroid node, and in the same way as above, we assign a path to $\mathcal{C}_{2}$ if it crosses a vertex from the subsets associated with these centroid nodes. Note that we only assign paths that were not assigned to the first class. Using the same arguments as above, we can compute an orientation that satisfies $\Omega\left(\left|\mathcal{C}_{2}\right| / k\right)$ requests. In particular, one can validate that each path crosses vertices from exactly one centroid node; otherwise, it should have been assigned to the first class by properties (2) and (3) of the tree decomposition. We now proceed recursively in the same way to construct the other classes as long as the decompositions under consideration are not empty. Since the maximal size of a subtree decreases by at least half in each level of the recursion, this process terminates within $\lceil\log \ell\rceil$ steps, and hence, there are indeed at most $\lceil\log \ell\rceil$ classes.

\section{A.2 Proof of Theorem 2.4}

The algorithm begins by finding a feedback vertex set $F=\left\{v_{1}, \ldots, v_{\ell}\right\}$ in the undirected version of the graph, namely, a set of vertices whose removal turns the underlying undirected graph into a tree. Although the computational task of finding a feedback vertex set with a minimum cardinality is NP-hard, there is a 2 -approximation algorithm for this problem $[5,4,7]$. Therefore, we may assume that the cardinality of that set satisfies $\ell \leq 2 k$. The algorithm proceeds by computing a shortest path $p_{i}$ for each request $\left(s_{i}, t_{i}\right) \in P$. Then, each path $p_{i}$ is classified into one of $\ell+1$ classes: if $p_{i}$ crosses the vertex $v_{j}$ and none of the vertices in $\left\{v_{1}, \ldots, v_{j-1}\right\}$ then it is assigned to class $\mathcal{C}_{j}$; otherwise, if $p_{i}$ does not cross any of the vertices of $F$, then it is assigned to class $\mathcal{C}_{\ell+1}$. Notice that we can satisfy $\Omega\left(\left|\mathcal{C}_{j}\right|\right)$ paths from any class $j$ by applying Theorem 2.2. Also notice that by deleting the vertices of $F$ from the graph $G$, we obtain a mixed graph which is a forest of trees, and all the paths in $\mathcal{C}_{\ell+1}$ still remain connected. This mixed tree orientation setting is known to admit an efficient $\Omega(1 / \log n)$-approximation algorithm [10], and thus, we can satisfy $\Omega\left(\left|\mathcal{C}_{\ell+1}\right| / \log n\right)$ paths from the class $\ell+1$. One can now easily validate that executing the mentioned algorithms on each class separately, and then picking the option that satisfies the highest number of paths results in an orientation satisfying $\Omega(|P| /(k+\log n))$ of all requests. 


\section{A.3 Proof of Theorem 2.5}

The algorithm computes a shortest path $p_{i}$ for each request $\left(s_{i}, t_{i}\right) \in P$. Then, it considers those shortest paths in some arbitrary order, and orients them one after the other. In particular, when a path $p$ is oriented, all the pending paths that are in conflict with $p$ are discarded. This greedy orientation procedure continues as long as a path $p$ under consideration is not in conflict with more than $\sqrt{\Delta|P|}$ pending paths. When this happens, there must be some vertex $v$ on $p$ that at least $\sqrt{\Delta|P|} / \Delta=\sqrt{|P| / \Delta}$ pending paths cross. This claim holds since the length of $p$ is known to be at most $\Delta$. The algorithm then employs the local-to-global orientation algorithm from Theorem 2.2 with respect to the vertex $v$ and the corresponding set of pending paths to complete the orientation of the graph.

One can easily verify that the algorithm computes a feasible orientation. Therefore, we next prove that the algorithm satisfies $\Omega(1 / \sqrt{\Delta|P|})$-fraction of all requests. Notice that if the algorithm employs our local-toglobal orientation algorithm then the resulting orientation indeed attains the desired performance guarantee since the number of paths satisfied only by this step is $\Omega(\sqrt{|P| / \Delta})=\Omega(1 / \sqrt{\Delta|P|}) \cdot|P|$. Hence, we may assume that the algorithm only makes greedy orientation steps. In this case, the resulting orientation clearly achieves the desired performance guarantee since one path is satisfied while at most $\sqrt{\Delta|P|}$ paths are discarded in each of those greedy steps. 